\title{
Proteinase-activated receptor 2 deficiency is a protective factor against cardiomyocyte apoptosis during myocardial ischemia/reperfusion injury
}

\author{
MIN WANG ${ }^{1 *}$, YIWEN MA ${ }^{2 *}$, TIANTIAN ZHANG ${ }^{1}$, LIN GAO $^{1}$, SHAN ZHANG $^{3}$ and QIZHI CHEN ${ }^{1}$ \\ Departments of ${ }^{1}$ Cardiology and ${ }^{2}$ Anesthesiology, Shanghai Ninth People's Hospital, \\ Shanghai Jiao Tong University School of Medicine, Shanghai 200011; ${ }^{3}$ Department of Emergency, \\ Tumor Hospital, Fudan University, Shanghai 200032, P.R. China
}

Received October 14, 2018; Accepted June 25, 2019

DOI: $10.3892 / \mathrm{mmr} .2019 .10618$

\begin{abstract}
Previous studies have established that proteinaseactivated receptor 2 (PAR2) activation protects against myocardial ischemia/reperfusion injury (MI/RI). However, the role of PAR2 deficiency in MI/RI remains unclear. The aim of the present study was to examine the effect of PAR2 deficiency on cardiomyocyte apoptosis and to clarify the potential molecular mechanisms for its protective effect against MI/RI. Using a mouse model of MI/RI, cardiac function was evaluated by echocardiography, infarct size was assessed by triphenyltetrazolium chloride staining, and myocardial cell apoptosis was measured by terminal deoxynucleotide transferase-mediated dUTP nick end-labeling staining. Annexin V/propidium iodide staining, and expression of Bcl-2 and cleaved PARP were determined to assess apoptosis in myocardial H9c2 cells exposed to hypoxia/reoxygenation (H/R) injury-simulating MI/RI. Phosphorylated ERK1/2, JNK, and p38 MAPK protein expression levels were analyzed by western blotting. The findings indicated that PAR2 deficiency markedly reduced cardiomyocyte apoptosis in the MI/RI mouse model, as well as in myocardial H9c2 cells exposed to H/R. Furthermore, PAR2 knockdown clearly prevented phosphorylation of ERK1/2 and JNK in myocardial H9c2 cells. The results revealed that PAR2 deficiency alleviated MI/RI-associated apoptosis by inhibiting phosphorylation of ERK $1 / 2$ and JNK. Therefore, targeted
\end{abstract}

Correspondence to: Dr Qizhi Chen, Department of Cardiology, Shanghai Ninth People's Hospital, Shanghai Jiao Tong University School of Medicine, 639 Zhi Zao Ju Road, Shanghai 200011, P.R. China

E-mail: nzhskzj@hotmail.com

*Contributed equally

Key words: proteinase-activated receptor 2, myocardial ischemiareperfusion injury, apoptosis, ERK1/2, JNK, MAPK
PAR2 silencing may be a potential therapeutic approach for alleviation of $\mathrm{MI} / \mathrm{RI}$.

\section{Introduction}

Myocardial infarction continues to be the primary cause of human morbidity and mortality worldwide (1). Accompanied by coronary blood flow recanalization, reperfusion may cause aggravated myocardial injury leading to infarct area enlargement, arrhythmia, and heart dysfunction, a phenomenon known as myocardial ischemia/reperfusion injury (MI/RI). Jennings et al (2) first described the phenomenon that cardiac myocyte necrosis was irreversible in the ischemic region and that ischemic cells were more severely injured after blood restoration in the dog myocardium. Nearly 2 million patients experience MI/RI under circumstances of acute myocardial infarction or sudden cardiac arrest each year (3). The underlying mechanisms for MI/RI include oxidative stress, calcium overload, apoptosis, and leukocyte accumulation (4). In view of the dreaded complication of reperfusion and the heavy social burden, it is urgently necessary to further explore the mechanism of MI/RI pathogenesis and to develop effective intervention measures.

Proteinase-activated receptor 2 (PAR2), a member of the $\mathrm{G}$ protein-coupled receptor superfamily, is widely distributed on cardiomyocytes, cardiac fibroblasts, vascular endothelial cells, inflammatory cells (neutrophils, monocytes, eosinophils, mast cells), epithelial cells, and vascular smooth muscle cells (5-7). After cleavage of the PAR2 N-terminus at a specific site (R36S37) by various serine proteases, including trypsin, mast cell tryptase, and coagulation factors VIIa and Xa (8-10), a new tethered ligand forms that triggers further receptor activation. Interactions of PAR2 with $G$ proteins or $\beta$-arrestin can initiate a variety of signaling cascade pathways. A previous study revealed that PAR2 activation induces an increase in intracellular calcium along with the production of IP3 and DAG (11). This pathway acts through activation of MAPKs that modulate several intracellular targets, including extracellular signal regulated kinase (ERK)1/2 and, to lesser extents, p38 and c-Jun $\mathrm{NH}_{2}$-terminal protein kinase (JNK) (12). Furthermore, PAR2 stimulation 
can elicit multiple responses to inflammation, angiogenesis, and infection as well as ischemic injury, which may be beneficial or detrimental depending on particular settings and even cell types.

As an environmentally sensitive receptor, there is a close relationship between PAR2 and MI/RI. In rats, PAR2 stimulation with agonist peptide (AP) before reperfusion inhibited cardiomyocyte apoptosis by upregulating $\mathrm{Bcl}-2$ and downregulating Bax (13). A previous study revealed that PAR2 deficiency decreased oxidative stress and inflammatory responses in a mouse model of MI/RI (14). However, the role of PAR2 deficiency in apoptosis during MI/RI has not been completely established. In the present study, the effect of PAR2 deficiency on cardiomyocyte apoptosis and the underlying mechanisms of its protective effect on MI/RI were determined, using a mouse model of MI/RI in vivo and $\mathrm{H} 9 \mathrm{c} 2$ cells as a model of hypoxia/reoxygenation $(\mathrm{H} / \mathrm{R})$ in vitro.

\section{Materials and methods}

Animals. C57BL/6J mice (wild-type) were purchased from the Experimental Animal Center of Shanghai Jiao Tong University School of Medicine and PAR2 knockout mice on the C57BL/6J background were purchased from The Jackson Laboratory. A total of 24 male mice (12-week-old; weight 20-22 g) were used for in vivo experiments, which were performed according to the National Institutes of Health Guide for the Care and Use of Laboratory Animals. Mice were housed in a specific pathogen-free (SPF) laboratory under optimum conditions $\left(25 \pm 2^{\circ} \mathrm{C}, 55 \%\right.$ humidity, and a $12 \mathrm{~h}$ light/dark cycle) and fed a standard laboratory diet and water ad libitum. The study procedures were approved by the Ethics Committee of Animal Experiments of Shanghai Jiao Tong University School of Medicine.

Genotyping of PAR2 knockout mice. For genotyping of mice, tail genomic DNA was isolated by using Mouse Direct PCR kit (cat. no. B40013; Bimake.com) according to the manufacturer's instructions. Then, the DNA lysates were subjected to PCR analysis with specific PAR2 primer. The primer sequences were as follows: oIMR7415, 5'-GCCAGAGGCCACTTGTGT AG-3'; oIMR7419, 5'-TCAAAGACTGCTGGTGGTTG-3'; and oIMR7420, 5'-GGTCCAACAGTAAGGCTGCT-3'; The PCR products were analyzed by $1 \%$ agarose gel electrophoresis performed by Gel image system 2500 .

Surgical procedures. A total of 24 mice were randomly divided into four groups as follows: the sham control mice in both PAR2 $2^{+/+}$and PAR2 $2^{-/}$groups (each $n=6$ ), the ischemia/reperfusion model in both PAR2 ${ }^{+/+}$and PAR2 ${ }^{-/}$groups (each $n=6)$. Mice were anesthetized with $2 \%$ isoflurane (Takeda Pharmaceutical Company, Ltd.) and $40 \%$ oxygen, and maintained with $0.5 \%$ isoflurane and $40 \%$ oxygen. A tracheal intubation was performed to provide artificial ventilation ( $0.3 \mathrm{ml}$ tidal volume; 120 breaths/min). The left anterior descending (LAD) coronary artery was ligated with an 8-0 nylon surgical suture at $1.0 \mathrm{~mm}$ distal from the tip of the left auricle. After $30 \mathrm{~min}$ of ligation, the suture was removed for reperfusion. Six sham control mice were subjected to the entire surgical procedure; however, sutures were passed through the LAD without blocking the vessels.

Measurements of infarct area and area at risk. After $120 \mathrm{~min}$ of reperfusion, mice were re-anesthetized and intubated. The heart was washed with normal saline via ascending aorta cannulation. The left coronary artery was reoccluded at the same point and Evans blue dye (cat. no. E2129; Sigma-Aldrich; Merck KGaA) was perfused from the left ventricular (LV) cavity. The heart was removed and cut transversely into five sections (2-mm thick) that were incubated in a $1.0 \%$ solution of triphenyltetrazolium chloride (TTC; cat. no. T8877; Sigma-Aldrich; Merck KGaA) for $20 \mathrm{~min}$ at $37^{\circ} \mathrm{C}$. The area at risk (AAR) and the infarct area (IA) were defined as the area unstained with Evans blue dye and the area unstained with TTC solution, respectively. Staining was quantified using Image 2 software (National Institutes of Health) as previously described (15). Infarct size was calculated as the percentage of myocardial IA to total AAR.

Apoptosis assessment using the TUNEL assay. In vivo TUNEL assays were performed to detect apoptotic cardiomyocytes induced by MI/RI $(16,17)$. Myocardial tissue was fixed with $10 \%$ formaldehyde for $24 \mathrm{~h}$, washed, dehydrated, and embedded in paraffin. TUNEL staining was carried out with an in situ Cell Death Detection Kit (cat. no. 11684817910; Roche Diagnostics) according to the manufacturer's instructions. Samples were added $50 \mu 1$ TUNEL reaction mixture in the dark at $37^{\circ} \mathrm{C}$ for $60 \mathrm{~min}$. Subsequently, the sections were counterstained with hematoxylin for a few seconds at $37^{\circ} \mathrm{C}$. Five microscopic fields in each section were randomly selected to count the number of TUNEL-positive cells and total cells. An apoptosis index (AI) score was calculated as the ratio of apoptotic cardiomyocytes to total myocytes.

Echocardiography. At $72 \mathrm{~h}$ after MI/RI, echocardiography was performed with a Vevo 770 high-resolution imaging system (VisualSonics Inc.) as previously described (18). Twelve mice were anesthetized and maintained in the supine position. After the chest was shaved, the parasternal short axis and long axis were examined to obtain two-dimensional and M-mode echocardiographic views. At least 10 independent cardiac cycles were acquired. The LV ejection fraction (LVEF) and LV fractional shortening (LVFS) were measured on M-mode images using the Vevo 770 software. The mice were sacrificed with an overdose of $10 \%$ chloral hydrate $(500 \mathrm{mg} / \mathrm{kg}$, i.v.), followed by cervical dislocation to minimize the suffering.

Cell culture. The rat cardiomyocyte cell line H9c2 was obtained from the Chinese Academy of Sciences Cell Bank (Shanghai, China). The cells were cultured in Dulbecco's modified Eagle's medium (cat. no. SH30022.01; HyClone; GE Healthcare Life Sciences) containing 10\% fetal bovine serum (cat. no. 10100147C; Gibco; Thermo Fisher Scientific, Inc.), $100 \mathrm{U} / \mathrm{ml}$ penicillin, and $100 \mathrm{mg} / 1$ streptomycin at $37^{\circ} \mathrm{C}$ in a humidified incubator under $5 \% \mathrm{CO}_{2}$.

siRNA transfection. Small interfering RNAs (siRNAs) against PAR2 (siPAR2) and a non-specific siRNA (siNC) were designed and synthesized (Shanghai GenePharma Co., 
Table I. Sequences of siRNAs used in the present study.

Genes

Sequences

Negative control (si-NC)

Sense: 5'-UUCUCCGAACGUGUCACGUTT-3'

Antisense: 5'-ACGUGACACGUUCGGAGAATT-3'

si-PAR2-257

Sense: 5'-CAGGCUUUUCCGUUGAUGATT-3'

Antisense: 5'-UCAUCAACGGAAAAGCCUGTT-3'

si-PAR2-566

Sense: 5'-ACUGCUCCAUCCUUUUCAUTT-3'

Antisense: 5'-AUGAAAAGGAUGGAGCAGUTT-3'

si-PAR2-1002

Sense: 5'-GCUGCUCGUCGUGCAUUAUTT-3'

Antisense: 5'-AUAAUGCACGACGAGCAGCTT-3'
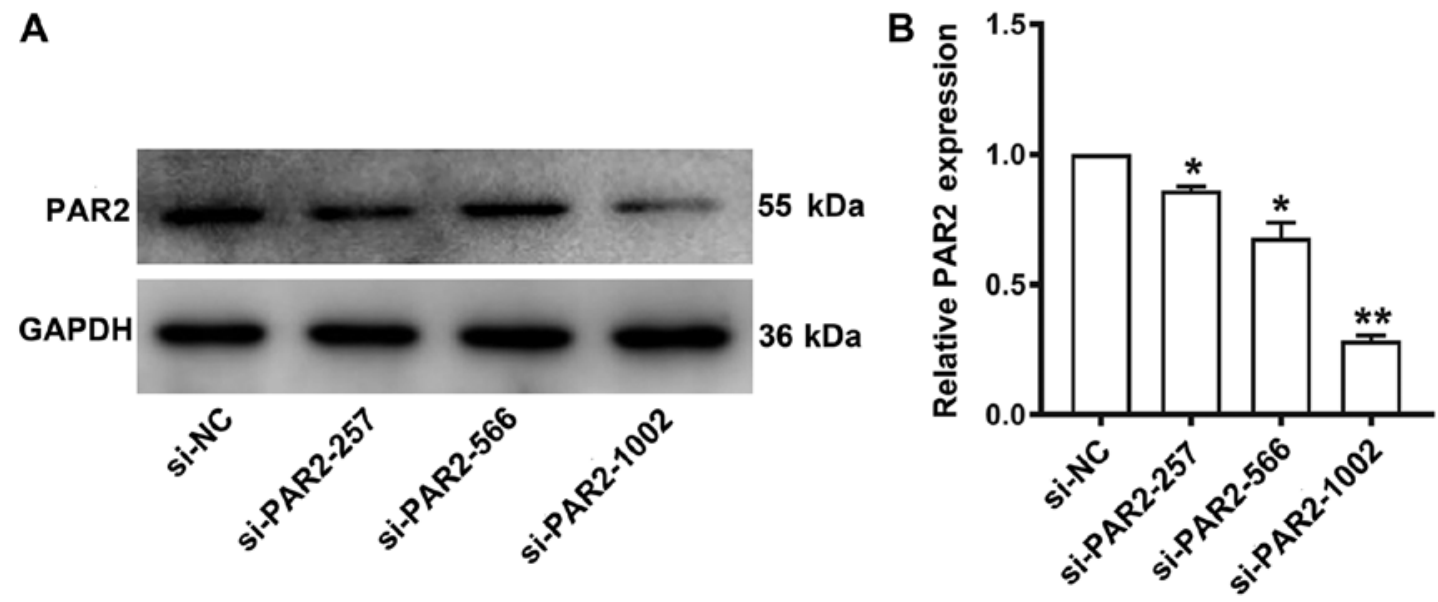

Figure 1. Efficiency of PAR2 knockdown in H9c2 cells. H9c2 cells were transfected with a PAR2 siRNA (si-PAR2) or non-specific siRNA (si-NC). (A) Knockdown efficiency evaluated by western blot analyses. (B) Densitometric analyses of the antibody-bound protein bands in the western blot analyses ( $\mathrm{P}<0.05$ and ${ }^{* *} \mathrm{P}<0.01$ compared to si-NC). PAR2, proteinase-activated receptor 2 .

Ltd.). The sequence information for the siRNAs is provided in Table I. For transient silencing of PAR2, H9c2 cells seeded on day $1\left(1 \times 10^{5}\right.$ or $1 \times 10^{6}$ cells/well) were subjected to two 6 -h transfection rounds on days 2 and 3 with $50 \mathrm{nM}$ siPAR2 and Lipofectamine 3000 transfection reagent (cat. no. L3000015; Thermo Fisher Scientific, Inc.) as previously described (19). siNC was transfected in parallel. The knockdown efficiency of PAR2 was $\sim 70 \%$ with siPAR2-rat-1002 (Fig. 1A and B). Thus, this sequence (PAR2-rat-1002) was selected to perform subsequent RNAi experiments.

Hypoxia and reoxygenation. To evaluate the effect of PAR2 deficiency on apoptosis, $\mathrm{H} 9 \mathrm{c} 2$ cells were transiently transfected for $48 \mathrm{~h}$, exposed to hypoxia $\left(94 \% \mathrm{~N}_{2}+5 \% \mathrm{CO}_{2}+1 \% \mathrm{O}_{2}\right)$ for $12 \mathrm{~h}$, and returned to normoxia $\left(95 \%\right.$ air $\left.+5 \% \mathrm{CO}_{2}\right)$ for $4 \mathrm{~h}$ (20). Cell viability was determined using a Cell Counting Kit-8 (cat. no. CK04; Dojindo Molecular Technologies, Inc.) according to the manufacturer's protocol.

Apoptosis assay. Cardiomyocyte apoptosis in vitro was estimated by flow cytometry after staining with Annexin V and propidium iodide (PI) (21). Briefly, cells were harvested and stained with an Annexin V-FITC/PI Kit (cat. no. 556547; BD Biosciences), in which Annexin $\mathrm{V}$ bound to exposed phosphatidylserine in early apoptotic cells and PI stained late apoptotic cells with increased membrane permeability. For the staining, cells were incubated in $5 \mu \mathrm{l}$ of Annexin $\mathrm{V}$ at room temperature in the dark for $10 \mathrm{~min}$, and then incubated with PI for an additional $5 \mathrm{~min}$. The Annexin-V/PI-stained cells were analyzed by flow cytometry (BD Biosciences).

Western blotting. Western blot analyses were carried out to measure the expression levels of multiple proteins in $\mathrm{H} 9 \mathrm{c} 2$ cells. The protocol was performed as described (22). Briefly, extracted proteins were separated in $10 \%$ SDS-polyacrylamide gels and transferred onto nitrocellulose membranes. The membranes were blocked with $5 \%(\mathrm{w} / \mathrm{v})$ nonfat dry milk in Tris-buffered saline for $2 \mathrm{~h}$ at room temperature, and incubated with the respective primary antibodies at $4^{\circ} \mathrm{C}$ overnight. The antibodies used were as follows: phospho-MAPK, total-MAPK, (phospho cat. no. 9910; 1:1,000; total cat. no. 9926, 1:1,000), poly-ADP-ribose polymerase (PARP; cat. no. 9532; 1:1,000), B-cell lymphoma 2 (Bcl-2; cat. no. 3498; 1:1,000 (23) (all from Cell Signaling Technology, Inc.); PAR2 (cat. no. 180953, 1:1,000; Abcam). After incubation with horseradish peroxidase-conjugated secondary antibodies Peroxidase AffiniPure Goat Anti-Rabbit IgG $(\mathrm{H}+\mathrm{L})$ (product 
code no. 111-035-003; 1:5,000; Jackson ImmunoResearch Laboratories, Inc.) at room temperature for $2 \mathrm{~h}$. GAPDH was evaluated as a loading control. Antibody-bound bands were detected with an enhanced chemiluminescence (ECL) detection kit (EMD Millipore). Quantification was performed using Quantity One 4.4.0 software (Bio-Rad Laboratories, Inc.).

Statistical analysis. All tests were repeated at least three times, and all data were expressed as the means \pm standard deviation. Statistical comparisons between mean values in multiple groups were evaluated by the Kolmogorov-Smirnov test, followed by one-way analysis of variance (ANOVA) using SPSS software (version 21.0; IBM Corp.). The Bonferroni test was performed to analyze multiple comparisons. Values of $\mathrm{P}<0.05$ were considered to indicate statistical significance.

\section{Results}

Successful identification of PAR2-knockout mice. The genotyping was confirmed in male PAR2 knockout mice by tail DNA genomic PCR. DNA from PAR2-knockout mice generated a 198-bp mutant band. As revealed in Fig. 2, a single PCR product of $198 \mathrm{bp}$ was amplified from the tail of PAR2-knockout mice. The results indicated that the generation of PAR2-knockout mice was successful for subsequent experiments.

PAR2 knockout preserves cardiac function after MI/RI in mice. To investigate ventricular function after MI/RI in mice, in vivo echocardiography was performed at $72 \mathrm{~h}$ after surgery. There were no significant differences in LVEF (72.3 \pm 9.7 vs. $69.8 \pm 4.1 \%, \mathrm{P}>0.05)$ and LVFS (39.3 \pm 5.4 vs. $37.8 \pm 3.5 \%, \mathrm{P}>0.05$ ) (Fig. 3 ) in the control PAR2 ${ }^{+/+}$group and $\mathrm{PAR}^{-/-}$group. MI/RI induced significant reductions in LVEF and LVFS in the two groups. Compared with PAR2 ${ }^{+/+}$mice, $\mathrm{PAR}^{-/-}$mice showed significant protective effects on LVEF $(47.8 \pm 2.3$ vs. $44.1 \pm 3.3 \%, P=0.047)$ and LVFS (25.4 \pm 1.2 vs. $21.0 \pm 2.4 \%, \mathrm{P}=0.002$ ) (Fig. 3). Overall, PAR2 knockout significantly improved LV function in mice after MI/RI.

PAR2 knockout reduces myocardial infarct size induced by $M I / R I$. To determine whether PAR 2 knockout attenuates myocardial damage after MI/RI, whole heart tissue was removed after surgery and stained. Infarcted myocardium was detected in both the PAR2 ${ }^{+/+}$and PAR2 ${ }^{-/}$groups. The ratio of AAR to LV did not differ in the two groups $(54.0 \pm 5.9$ vs. $45.4 \pm 10.7 \%, \mathrm{P}=0.115)$, however the ratio of IA to $\mathrm{LV}$ was reduced in the PAR2 ${ }^{-1}$ group (35.4 \pm 5.7 vs. $24.9 \pm 8.7 \%, \mathrm{P}=0.032$ ) (Fig. 4). As a result, $\mathrm{PAR}^{-/-}$mice had less infarct tissue compared with PAR2 ${ }^{+/+}$ mice $(16 \%$ reduction in infarct size; $55.41 \pm 7.3$ vs. $65.60 \pm 6.7 \%$, $\mathrm{P}=0.030$; Fig. 4). The reduction in myocardial infarct size along with the improvement in cardiac function indicated that PAR2 knockout had a protective effect against MI/RI.

PAR2 knockout protects against myocardial cell apoptosis induced by $M I / R I$. Cardiomyocyte apoptosis caused by MI/RI is one of the main causes of cardiac dysfunction (24). To investigate whether PAR2 has a role in the apoptotic process under MI/RI, TUNEL assays were performed to detect apoptotic

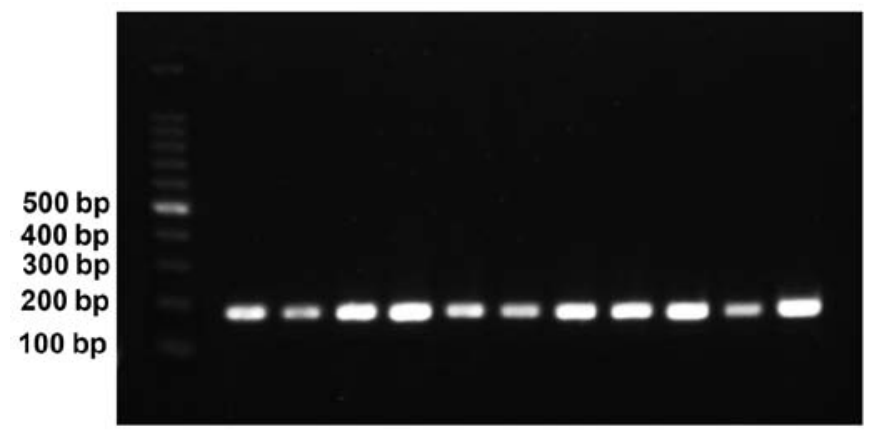

Figure 2. Result of PAR2-knockout mouse PCR amplification. PAR2 knockout mice generated a single PCR product of $198 \mathrm{bp}$. PAR2, proteinase-activated receptor 2 .

myocardium in the two groups. Apoptotic cells were revealed in the myocardium after MI/RI in both groups, and were mainly located in the area of the left anterior descending coronary artery territory. A $16 \%$ reduction in apoptotic cells was detected in PAR2 $2^{-/-}$mice compared with PAR2 $2^{+/+}$mice (56.3 \pm 2.2 vs. $66.5 \pm 3.7 \%, \mathrm{P}=0.005$; Fig. 5 ).

PAR2 knockdown protects against myocardial H9c2 cell apoptosis induced by $H / R$. To further validate the protective effects of PAR2 knockout against myocardial cell apoptosis in MI/RI, RNA interference methods were applied in the rat cardiomyocyte line H9c2. H9c2 cells were pretreated with siPAR2 or siNC and then subjected to H/R. Cardiomyocyte apoptosis was evaluated by Annexin V/PI flow cytometric assay. The percentage of early apoptotic cells (Annexin $\mathrm{V}^{+} / \mathrm{PI}^{-}$) was significantly decreased in the PAR2 ${ }^{-/} \mathrm{H} / \mathrm{R}$ group compared with the PAR2 $2^{+/+}$ $\mathrm{H} / \mathrm{R}$ group (4.8 \pm 0.6 vs. $8.9 \pm 1.5 \%, \mathrm{P}=0.003$; Fig. 6 ).

Next, the relative levels of apoptosis-related proteins such as cleaved PARP (c-PARP) and Bcl-2 were analyzed by western blotting. PAR2 knockdown led to inhibition of PARP cleavage $(3.4 \pm 0.5$ vs. $6.6 \pm 0.4, \mathrm{P}<0.001)$ and stimulation of $\mathrm{Bcl}-2$ expression $(8.2 \pm 0.4$ vs. $4.3 \pm 0.4, \mathrm{P}<0.001)$ in the PAR2 $2^{-/} \mathrm{H} / \mathrm{R}$ group compared with the PAR $2^{+/+} \mathrm{H} / \mathrm{R}$ group (Fig. 7). Consistent with the TUNEL staining in vivo, both the Annexin V/PI staining observations and expression levels of apoptotic proteins (c-PARP and Bcl-2) indicated that H/R-induced apoptosis was decreased by PAR 2 knockdown.

PAR2 knockdown inhibits ERK1/2 and JNK pathways in myocardial $H 9 c 2$ cells exposed to $H / R$. To further understand the potential mechanisms for the PAR2 protective role in MI/RI, western blot analyses were performed to quantify the MAPK phosphorylation levels in H9c2 cells exposed to $\mathrm{H} / \mathrm{R}$. The PAR $2^{-{ }_{-}} \mathrm{H} / \mathrm{R}$ group exhibited significantly decreased phosphorylation levels of ERK1/2 (1.23 \pm 0.02 vs. $1.55 \pm 0.02$, $\mathrm{P}<0.001)$ and JNK $(0.79 \pm 0.03$ vs. $1.28 \pm 0.04, \mathrm{P}<0.001)$ compared with the PAR2 ${ }^{+/+} \mathrm{H} / \mathrm{R}$ group, while the phosphorylation levels of p38 MAPK did not differ $(\mathrm{P}>0.05)$ between the groups (Fig. 8).

\section{Discussion}

In the present study, it was confirmed that PAR2 deficiency protected the myocardium against apoptosis using an in vivo 
A
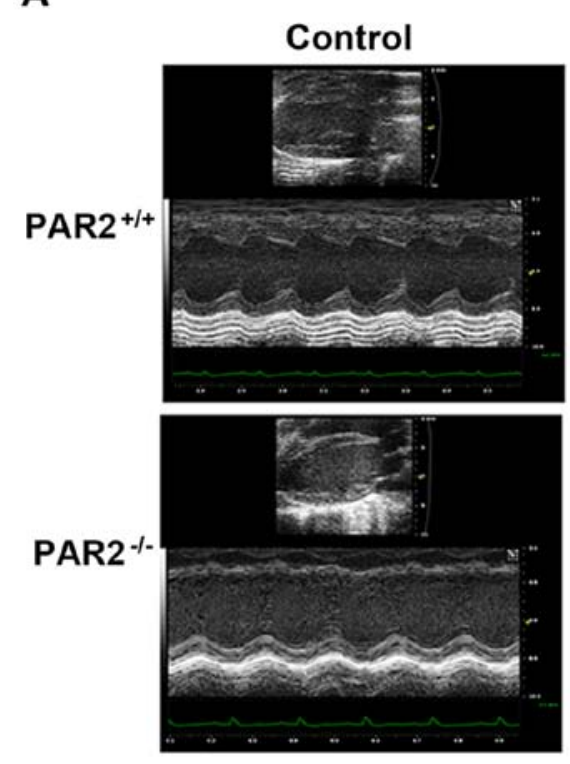

$\mathbf{I} / \mathbf{R}$
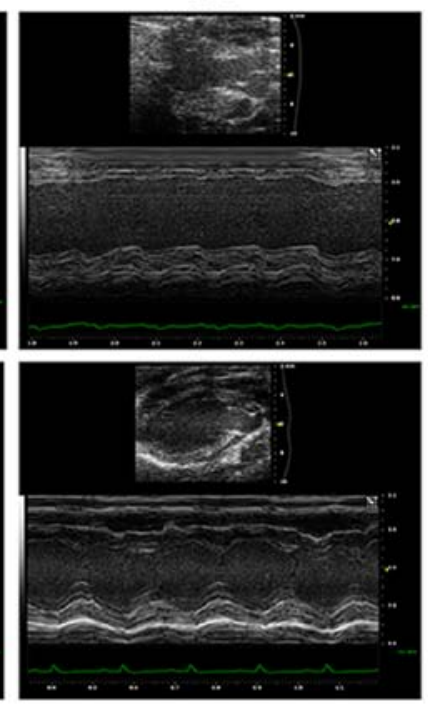

B
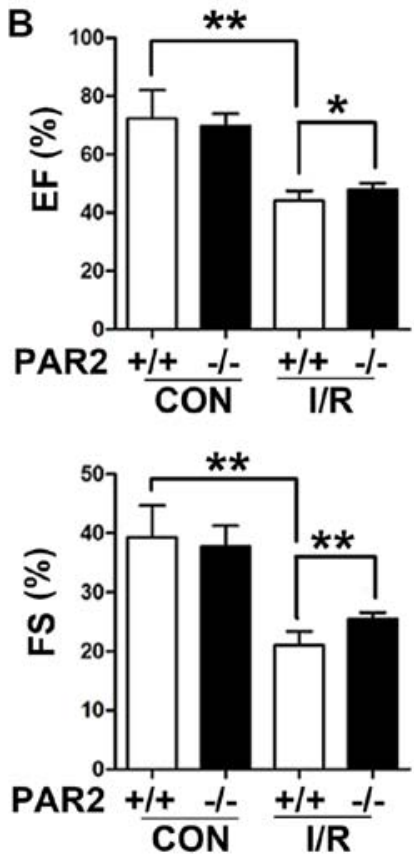

Figure 3. Effects of PAR2 knockout on cardiac function after MI/RI in vivo. (A) Representative echocardiographs of mice after MI/RI. (B) Percentages of LVEF and LVFS after MI/RI ( ${ }^{*} \mathrm{P}<0.05$ and $\left.{ }^{* *} \mathrm{P}<0.01\right)$. PAR2, proteinase-activated receptor 2; MI/RI, myocardial ischemia/reperfusion injury; LV, left ventricular; LVEF, LV ejection fraction; LVFS, LV fractional shortening.

A

PAR2 $^{+/+}$

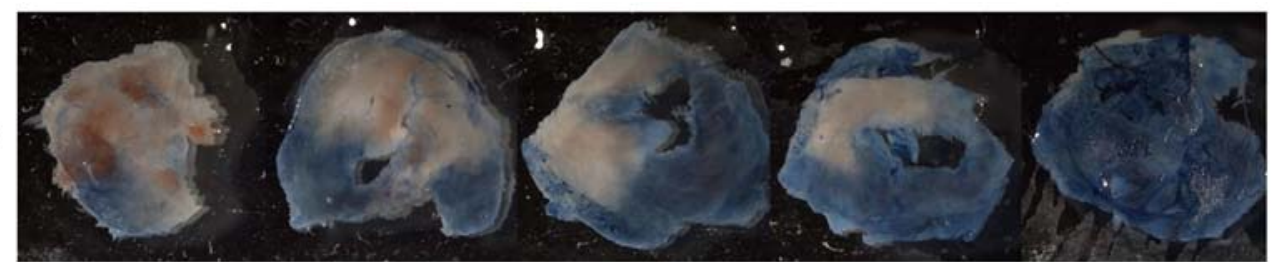

PAR2 $^{-1}$

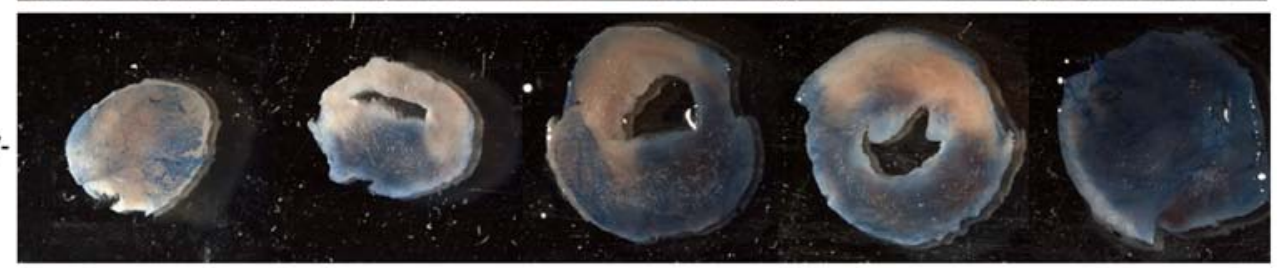

B
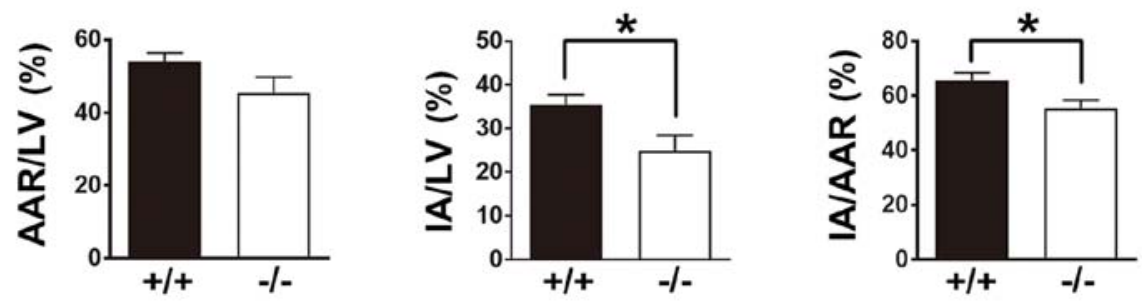

Figure 4. IA and AAR data in the LV after MI/RI. (A) Representative Evans Blue-stained images of the myocardium in PAR2 $2^{+/+}$mice and PAR2 ${ }^{-/}$mice (B) Summarized data for ratios of AAR to LV, IA to LV and IA to AAR in PAR2 $2^{+/+}$mice and PAR2 $2^{-/}$mice ("P $\left.<0.05\right)$. AAR, area at risk; IA, infarct area; $\mathrm{LV}$, left ventricular; MI/RI, myocardial ischemia/reperfusion injury; PAR2, proteinase-activated receptor 2.

MI/RI mouse model. It was further revealed that PAR2 knockdown in $\mathrm{H} 9 \mathrm{c} 2$ cells caused a significant reduction in apoptosis accompanied by downregulation of c-PARP expression and upregulation of Bcl-2 expression. Reductions in ERK and JNK phosphorylation were also observed, suggesting that PAR2 deficiency may protect the myocardium against MI/RI via MAPK-dependent anti-apoptotic effects on cardiomyocytes. Persistent ischemia and reperfusion can induce cardiomyocyte apoptosis, especially after reperfusion, through sudden recovery of intracellular ATP levels, calcium overload in the cytoplasm and mitochondria, and production of oxygen free radicals that can further accelerate irreversible apoptosis (3). Many studies have revealed that apoptosis may be a significant factor in the pathogenesis of MI/RI. Apoptosis is a genetically-controlled cell death process that primarily requires the activation of caspases. In fact, PARP cleavage by caspase- 3 is considered a 

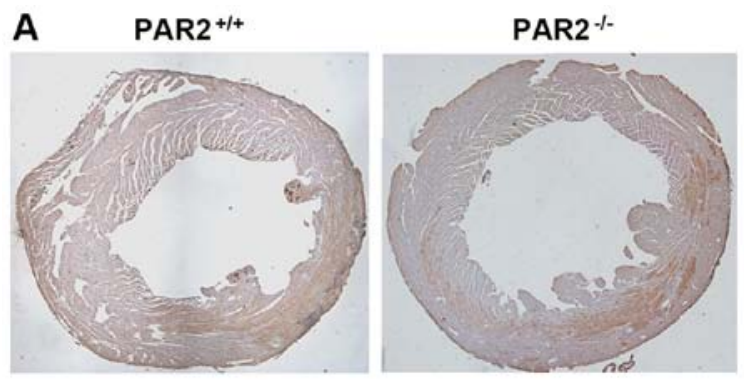

B

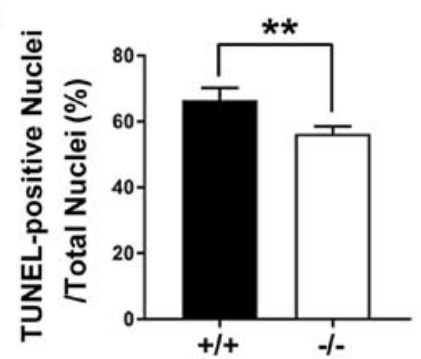

Figure 5. Apoptosis of cardiac myocytes is decreased in PAR2 ${ }^{-/}$mice after MI/RI. (A) Representative photomicrographs of TUNEL-stained myocardial sections from PAR2 $2^{+/}$mice and PAR2 $2^{-/}$mice ( $\mathrm{x} 40$ magnification). (B) Quantification of mean AI scores obtained in the TUNEL assays $\left({ }^{* *} \mathrm{P}<0.01\right)$. PAR2, proteinase-activated receptor 2; MI/RI, myocardial ischemia/reperfusion injury; TUNEL, terminal deoxynucleotide transferase-mediated dUTP nick end-labeling; AP, apoptosis index.

A

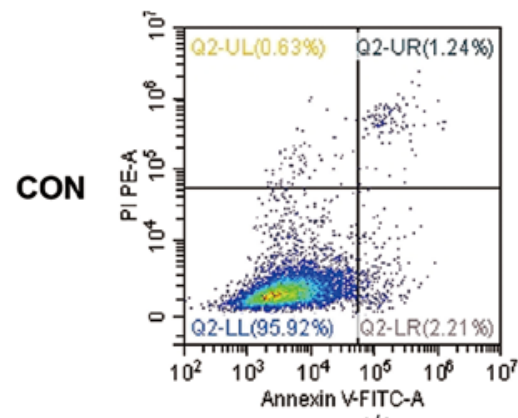

PAR2 $^{+1+}$

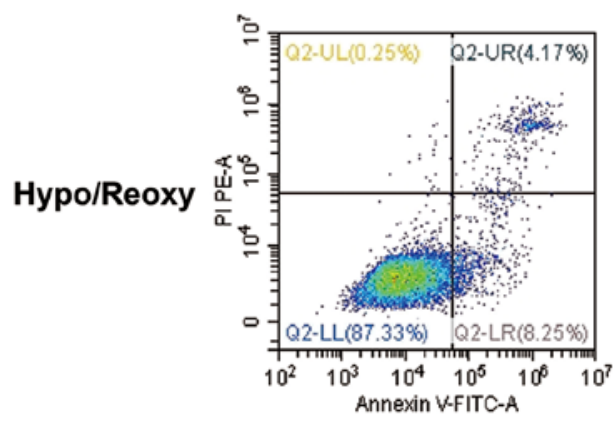

PAR2 $^{+/+}$

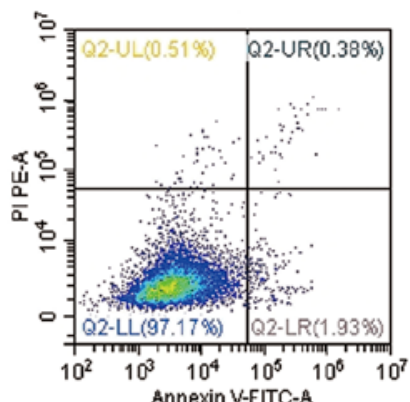

PAR2 ${ }^{-1}$

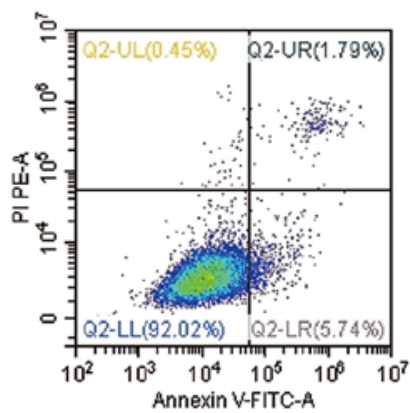

PAR2 1 -
B

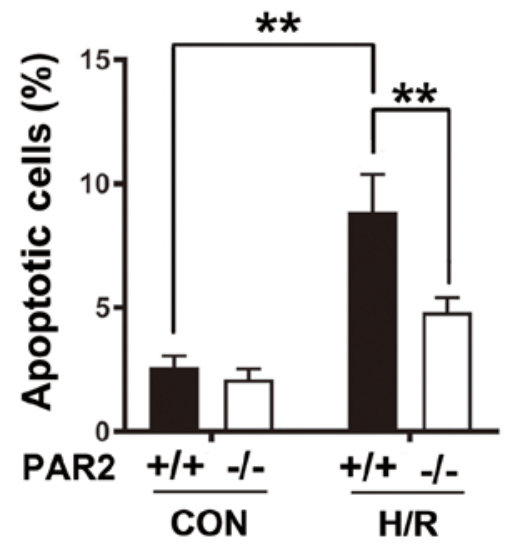

Figure 6. Effects of PAR2 knockdown on percentages of apoptotic H9c2 cells. (A) After H/R treatment, H9c2 cells were stained with FITC-Annexin V and PI and evaluated by flow cytometry. (B) The percentages of Annexin $\mathrm{V}^{+} / \mathrm{PI}{ }^{-}$cells were analyzed $\left({ }^{* *} \mathrm{P}<0.01\right)$. PAR2, proteinase-activated receptor $2 \mathrm{PI}$, propidium iodide; $\mathrm{H} / \mathrm{R}$, hypoxia/reoxygenation.

A

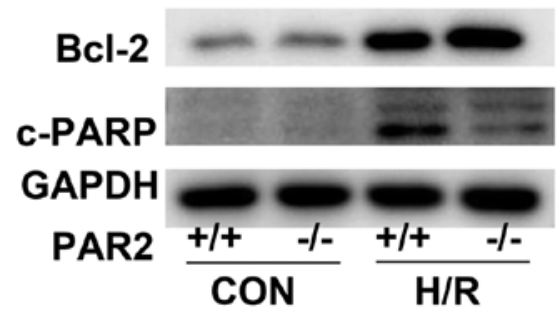

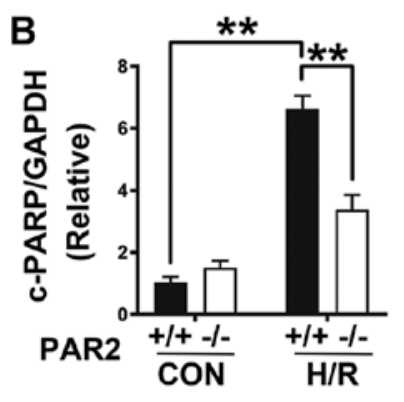

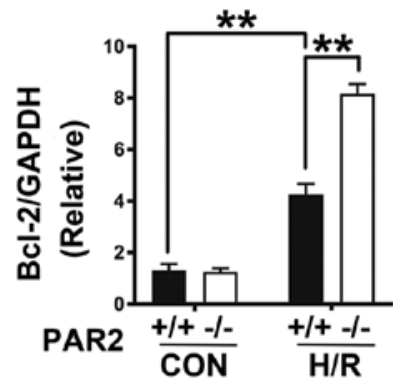

Figure 7. Effects of PAR2 knockdown on apoptosis-associated molecules. (A) Western blot analyses of cleaved PARP and Bcl-2 in the indicated groups. (B) Densitometric analyses of the antibody-bound protein bands in the western blotting analyses $\left({ }^{* *} \mathrm{P}<0.01\right)$. PAR2, proteinase-activated receptor 2 ; PARP, poly-ADP-ribose polymerase; Bcl-2, B-cell lymphoma 2.

marker for the apoptotic cell death process. Excessive activation of PARP exacerbates apoptosis by exhausting the amounts of cellular nicotinamide adenine dinucleotide and adenosine triphosphate (25). The Bcl-2 family members (Bcl-2, Bcl-xL, 
A

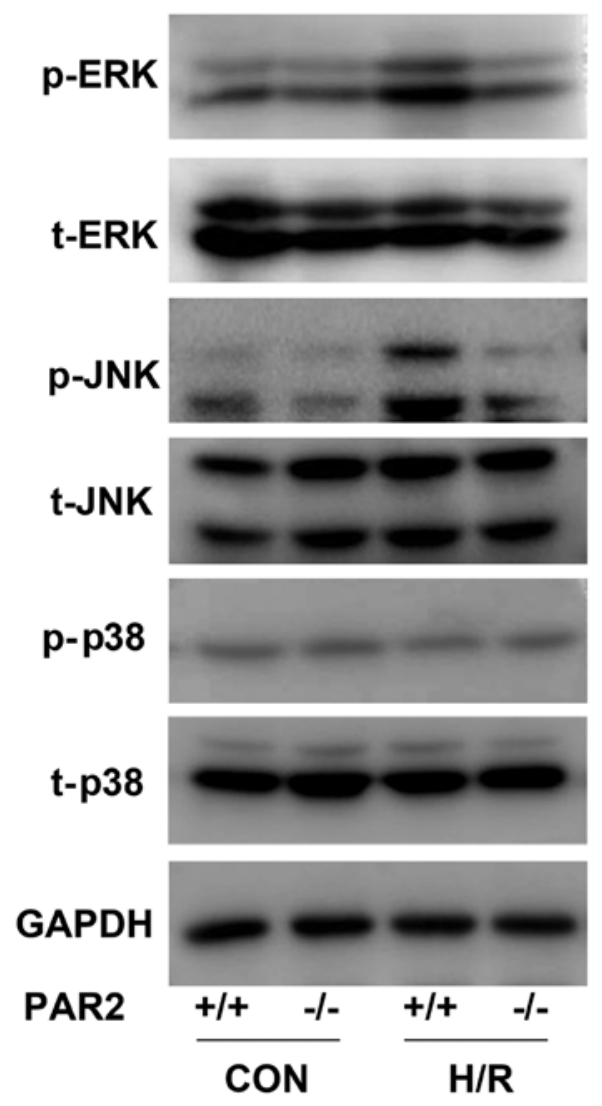

B
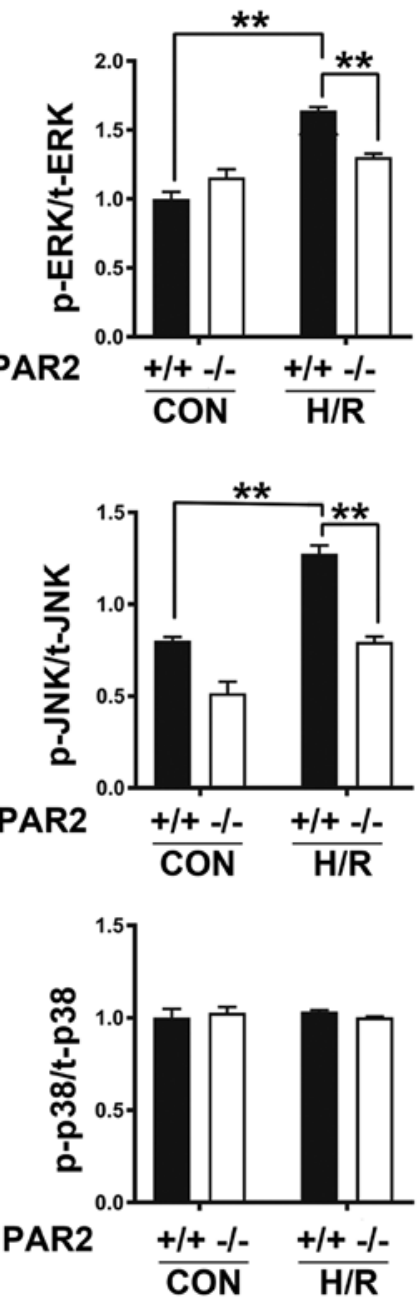

Figure 8. Effects of PAR2 knockdown on MAPK signaling pathways in H9c2 cells after H/R injury. (A) Representative western blotting images for phosphorylation levels of ERK1/2, JNK, and p38 MAPK. (B) Densitometric analyses of antibody-bound protein bands in the western blot analyses (**P<0.01). PAR2, proteinase-activated receptor 2; H/R, hypoxia/reoxygenation; ERK, extracellular signal regulated kinase; JNK, c-Jun NH2-terminal protein kinase.

Bax, Bak) are involved in the regulation of apoptotic signaling by mediating mitochondrial membrane permeability. Bcl-2 plays a vital role in the anti-apoptosis process by inhibiting Bax migration to the mitochondrial membrane or interfering with the Bak oligomerization process (26). The present results demonstrated that PAR2 deficiency attenuated cardiomyocyte apoptosis after H/R with decreased c-PARP and increased Bcl-2 expression levels.

The molecular targets for the PAR2 deficiency-mediated protective effects against cardiomyocyte apoptosis in MI/RI were further investigated, especially with regard to the MAPK signaling pathways, for the following reasons. First, it was reported that PAR2 can activate MAPKs in the myocardium and other tissues $(27,28)$. PAR2 couples to $G$ proteins (Gaq, $\mathrm{G} \alpha \mathrm{i}, \mathrm{G} \alpha \mathrm{s}, \mathrm{G} \alpha 12 / 13$ ) as well as $\beta$-arrestin to induce downstream signaling. The Goi pathway predominantly activates the MAPK pathways, while activation of $\beta$-arrestin leads to phosphorylation of ERK1/2 (9,29). Activation of PAR2 was revealed to have protective effects on the myocardium via the ERK1/2 pathway during reperfusion of the rat heart (30). Antoniak et al (14) demonstrated that PAR2 deficiency alleviates myocardial inflammation accompanied by reduced phosphorylation of ERK1/2, p38, and JNK after MI/RI.
Furthermore, they found that PAR2 activation stimulated hypertrophy of cardiomyocytes in vitro with increased phosphorylation of p38 and ERK1/2 (31). Second, it was revealed that MAPK signaling pathways, including the ERK and JNK pathways, were involved in the regulation of myocardial apoptosis during MI/RI and H/R (32). ERK signaling plays major roles in cell survival and differentiation, being cytoprotective in most cases (33). However, other studies have revealed that enhanced ERK activation promoted apoptosis. H/R caused cardiomyocyte apoptosis associated with enhanced phosphorylation of ERK1/2 (34). ERK activation also contributed to cisplatin-induced cell apoptosis, while cell death was attenuated after inhibition of ERK phosphorylation by a specific inhibitor (PD98059) (35). JNK activation mediated inflammation and apoptosis in response to stress stimuli (33). JNK activation was enhanced during MI/RI as a mediator of the apoptotic pathway. Inhibition of JNK by SP600125 improved cardiac function, and still prevented cardiomyocyte apoptosis in the rat heart after MI/RI (36). In the present study, it was revealed that PAR2 deficiency alleviated MI/RI by decreasing cardiomyocyte apoptosis through suppression of the ERK and JNK MAPK pathways, but not the p38 MAPK pathway. 
It was previously demonstrated that activation of PAR 2 had a positive effect on experimental MI/RI in vivo. Napoli et al (37) were the first to demonstrate that PAR2 AP treatment improved recovery of myocardial function and mitigated oxidative injury in wild-type rats through reductions in the ischemic risk zone and creatine kinase release. In contrast to the benefit of PAR2 activation, PAR2 deficiency had protective effects on cardiac $\mathrm{MI} / \mathrm{RI}$ with significant reductions in oxidative stress and inflammation (14). Similar results were revealed in the lungs and joints $(38,39)$. A possible explanation for the discrepancies may be attributed to cell specificity. In the MI/RI model, PAR2 may exert a protective effect when activated on endothelial cells by exogenous PAR2 AP (40), but may be harmful when activated on cardiomyocytes or inflammatory cells that are inclined to stimulate production of inflammatory factors (41). Thus, through their systemic lack of PAR2, inflammation and oxidative stress may be inhibited in PAR2 ${ }^{-/}$mice. Furthermore, a trypsin-exposed tethered ligand and a synthetic soluble ligand bound to different regions of PAR2, thereby triggering biased signaling. Exogenous PAR2 AP may act on distinct sites in PAR2, leading to markedly different effects. Therefore, it was speculated that the cytoprotective effect of PAR2 AP may be associated with the manner of PAR2-biased signaling. Finally, altered PAR2 conformations gave rise to $G$ protein-coupled changes and activation of $\beta$-arrestin, thereby mediating receptor desensitization and internalization (42). Another study demonstrated that $\beta$-arrestin alleviated inflammation by preventing phosphorylation and degradation of $\mathrm{I} \kappa \mathrm{B} \alpha$, and reducing activation of NF- $\kappa \mathrm{B}$ and transcription (43). In addition, $\beta$-arrestin transactivated epithelial growth factor receptor to generate a protective effect for the myocardium (44).

It was also demonstrated that PAR2 contributes to the occurrence and development of inflammation and oxidative stress. Although exogenous stimulation of PAR2 had a cardioprotective effect in MI/RI (37), the present results revealed that PAR2 deficiency decreased myocardial cell apoptosis in $\mathrm{MI} / \mathrm{RI}$, indicating that PAR2 activation by endogenous factors may have a detrimental role. Therefore, it is necessary to understand the exact role of PAR2 in MI/RI, and the precise molecular mechanisms and signal transduction pathways still require further clarification. Multiple cell types can be involved in MI/RI in different forms, including vascular endothelial cells, cardiomyocytes, and inflammatory cells/immunocytes. Therefore, the examination of tissue-specific PAR2 $2^{-/}$animals may be required to clarify the precise roles of PAR2 in different cells and to identify tissue-specific antagonists or agonists and even signaling pathways that target physiological or pathological events.

The limitation of the present study is that the methods used in the in vivo experiments in mice were relatively simple. However, our research methods used for the in vivo experiments of apoptosis including TUNEL analysis and AI count were based on previously published studies $(16,17)$. Second, the $\mathrm{H} 9 \mathrm{c} 2$ cell line was used, rather than examining neonatal mouse cardiomyocytes. However, it is worth pointing out that the $\mathrm{H} 9 \mathrm{c} 2$ cell line was obtained from an embryonic rat heart, and the cells are quite similar to cardiomyocytes. Third, no human data are currently available for the effects of PAR2 on $\mathrm{MI} / \mathrm{RI}$, and further studies are required for cardiomyocytes and cardiac tissues in humans. It is unrealistic to perform global knockout of PAR2 in human cardiomyocytes, and thus the development of selective and specific PAR2 antagonists or PAR2-blocking antibodies may be therapeutic strategies for the alleviation of MI/RI.

In conclusion, the present study demonstrated that PAR2 deficiency could protect the myocardium against MI/RI by inhibiting apoptosis in vivo and in vitro through the suppression of ERK and JNK MAPK phosphorylation. Therefore, targeted inhibition of PAR2 may provide new insights toward the clinical prevention and treatment of acute MI/RI.

\section{Acknowledgements}

Not applicable.

\section{Funding}

This work was supported by the National Natural Science Foundation of China [grant no. 8130087]; and the Joint Research Project of Major Diseases, Shanghai Municipal Commission of Health and Family Planning [grant no. 2014ZYJB0501].

\section{Availability of data and materials}

The datasets generated and analyzed during the study are available from the corresponding author on reasonable request.

\section{Authors' contributions}

QC, MW and YM conceived the experiments. MW and YM implemented the experiments. TZ, LG and SZ arranged and analyzed the data. MW and YM wrote the manuscript. QC, TZ, LG and SZ revised the manuscript. All authors read and approved the manuscript.

\section{Ethics approval and consent to participate}

The study procedures were approved by the Ethics Committee of Animal Experiments of Shanghai Jiao Tong University School of Medicine.

\section{Patient consent for publication}

Not applicable.

\section{Competing interests}

The authors declare that they have no competing interests.

\section{References}

1. Mozaffarian D, Benjamin EJ, Go AS, Anett DK, Blaha MJ, Cushman M, de Ferranti S, Despres JP, Fullerton HJ, Howard VJ, et al: Heart disease and stroke statistics-2015 update: A report from the American Heart Association. Circulation 131: e29-e322, 2015.

2. Jennings RB, Sommers HM, Smyth GA, Flack HA and Linn H: Myocardial necrosis induced by temporary occlusion of a coronary artery in the dog. Arch Pathol 70: 68-78, 1960.

3. Turer AT and Hill JA: Pathogenesis of myocardial ischemia-reperfusion injury and rationale for therapy. Am J Cardiol 106: $360-368,2010$ 
4. Wang Z, Wu G, Liu H, Xing N, Sun Y, Zhai Y, Yang B, Kong AT, Kuang $\mathrm{H}$ and Wang Q: Cardioprotective effect of the xanthones from Gentianella acuta against myocardial ischemia/reperfusion injury in isolated rat heart. Biomed Pharmacother 93: 626-635, 2017.

5. Rothmeier AS and Ruf W: Protease-activated receptor 2 signaling in inflammation. Semin Immunopathol 34: 133-149, 2012.

6. Steinberg SF: The cardiovascular actions of protease-activated receptors. Mol Pharmacol 67: 2-11, 2005.

7. Murray DB, McLarty-Williams J, Nagalla KT and Janicki JS: Tryptase activates isolated adult cardiac fibroblasts via protease activated receptor-2 (PAR-2). J Cell Commun Signal 6: 45-51, 2012.

8. Morris DR, Ding Y, Ricks TK, Gullapalli A, Wolfe BL and Trejo J: Protease-activated receptor-2 is essential for factor VIIa and Xa-induced signaling, migration, and invasion of breast cancer cells. Cancer Res 66: 307-314, 2006

9. Adams MN, Ramachandran R, Yau MK, Suen JY, Fairlie DP, Hollenberg MD and Hooper JD: Structure, function and pathophysiology of protease activated receptors. Pharmacol Ther 130: 248-282, 2011.

10. Yau MK, Liu L and Fairlie DP: Toward drugs for proteaseactivated receptor 2 (PAR2). J Med Chem 56: 7477-7497, 2013.

11. Bohm SK, Kong W, Bromme D, Smeekens SP, Anderson DC Connolly A, Kahn M, Nelken NA, Coughlin SR, Payan DG and Bunnett NW: Molecular cloning, expression and potential functions of the human proteinase-activated receptor-2. Biochem J 314: 1009-1016, 1996.

12. Kanke T, Macfarlane SR, Seatter MJ, Davenport E, Paul A, McKenzie RC and Plevin R: Proteinase-activated receptor-2-mediated activation of stress-activated protein kinases and inhibitory kappa B kinases in NCTC 2544 keratinocytes. J Biol Chem 276: 31657-31666, 2001.

13. Tong XH, Ding JW, Yang J, Liu CQ, Zhang Y, Li S, Li WH and Li L: The effect of protease-activated receptor 2 on rat apoptotic cardiomyocytes underwent ischemia reperfusion injury. Zhonghua xin xue guan bing za zhi (Chinese) 37: 832-836, 2009.

14. Antoniak S, Rojas M, Spring D, Bullard TA, Verrier ED, Blaxall BC, Mackman N and Pawlinski R: Protease-activated receptor 2 deficiency reduces cardiac ischemia/reperfusion injury. Arterioscler Thromb Vasc Biol 30: 2136-2142, 2010.

15. Rueden CT, Schindelin J, Hiner MC, DeZonia BE, Walter AE, Arena ET and Eliceiri KW: ImageJ2: ImageJ for the next generation of scientific image data. BMC Bioinformatics 18 : 529, 2017.

16. Odashima M, Usui S, Takagi H, Hong C, Liu J, Yokota M and Sadoshima J: Inhibition of endogenous Mstl prevents apoptosis and cardiac dysfunction without affecting cardiac hypertrophy after myocardial infarction. Circ Res 100: 1344-1352, 2007.

17. Chen PJ, Shang AQ, Yang JP and Wang WW: microRNA-874 inhibition targeting STAT3 protects the heart from ischemia-reperfusion injury by attenuating cardiomyocyte apoptosis in a mouse model. J Cell Physiol 234: 6182-6193, 2019.

18. Bunck AC, Engelen MA, Schnackenburg B, Furkert J, Bremer C Heindel W, Stypmann J and Maintz D: Feasibility of functional cardiac MR imaging in mice using a clinical 3 Tesla whole body scanner. Invest Radiol 44: 749-756, 2009.

19. Mußbach F, Ungefroren H, Günther B, Katenkamp K, Henklein P, Westermann M, Settmacher U, Lenk L, Sebens S, Müller JP, et al: Proteinase-activated receptor 2 (PAR2) in hepatic stellate cells-evidence for a role in hepatocellular carcinoma growth in vivo. Mol Cancer 15: 54, 2016.

20. Gu J, Fan Y, Liu X, Zhou L, Cheng J, Cai R and Xue S: SENP1 protects against myocardial ischaemia/reperfusion injury via a HIF1 $\alpha$-dependent pathway. Cardiovasc Res 104: 83-92, 2014.

21. Liang S, Aiqun M, Figtree G and Ping Z: GAPDH-silence preserves $\mathrm{H} 9 \mathrm{C} 2$ cells from acute hypoxia and reoxygenation injury. Int J Biol Macromol 81: 375-386, 2015.

22. Iablokov V, Hirota CL, Peplowski MA, Ramachandran R, Mihara K, Hollenberg MD and MacNaughton WK: Proteinaseactivated receptor 2 (PAR2) decreases apoptosis in colonic epithelial cells. J Biol Chem 289: 34366-34377, 2014.

23. Li W, Liu Y, Wang B, Luo Y, Hu N, Chen D, Zhang X and Xiong Y: Protective effect of berberine against oxidative stress-induced apoptosis in rat bone marrow-derived mesenchymal stem cells. Exp Ther Med 12: 4041-4048, 2016.

24. Dong Q, Li J, Wu QF, Zhao N, Qian C, Ding D, Wang BB, Chen L, Guo KF, Fu D, et al: Blockage of transient receptor potential vanilloid 4 alleviates myocardial ischemia/reperfusion injury in mice. Sci Rep 7: 42678, 2017.
25. Meng X, Song W, Deng B, Xing Z and Zhang W: 3-aminobenzamide, one of poly(ADP-ribose)polymerase-1 inhibitors, rescuesapoptosisin rat models of spinal cord injury. Int J Clin Exp Pathol 8: 12207-12215, 2015

26. Badr R, Hashemi M, Javadi G, Movafagh A and Mahdian R Assessment of global ischemic/reperfusion and Tacrolimus administration on CA1 region of hippocampus: Gene expression profiles of BAX and BCL2 genes. Bratisl Lek Listy 117: 358-362, 2016.

27. Sabri A, Muske G, Zhang H, Pak E, Darrow A, AndradeGordon P and Steinberg SF: Signaling properties and functions of two distinct cardiomyocyte protease-activated receptors. Circ Res 86: 1054-1061, 2000.

28. Darmoul D, Gratio V, Devaud H and Laburthe M: Proteaseactivated receptor 2 in colon cancer: Trypsin-induced MAPK phosphorylation and cell proliferation are mediated by epidermal growth factor receptor transactivation. J Biol Chem 279: 20927-20934, 2004.

29. Katritch V, Cherezov V and Stevens RC: Structure-function of the G protein-coupled receptor superfamily. Annu Rev Pharmacol Toxicol 53: 531-556, 2013.

30. Jiang R, Zatta A, Kin H, Wang N, Reeves JG, Mykytenko J, Deneve J, Zhao ZQ, Guyton RA and Vinten-Johansen J: PAR-2 activation at the time of reperfusion salvages myocardium via an ERK1/2 pathway in in vivo rat hearts. Am J Physiol Heart Circ Physiol 293: H2845-H2852, 2007.

31. Antoniak S, Sparkenbaugh EM, Tencati M, Rojas M, Mackman N and Pawlinski R: Protease activated receptor-2 contributes to heart failure. PLoS One 8: e81733, 2013.

32. Yu D, Li M, Tian Y, Liu J and Shang J: Luteolin inhibits ROS-activated MAPK pathway in myocardial ischemia/reperfusion injury. Life Sci 122: 15-25, 2015.

33. Portbury AL, Ronnebaum SM, Zungu M, Patterson C and Willis MS: Back to your heart: Ubiquitin proteasome system-regulated signal transduction. J Mol Cell Cardiol 52: 526-537, 2012.

34. Sun L, Fan H, Yang L, Shi L and Liu Y: Tyrosol prevents ischemia/reperfusion-induced cardiac injury in H9c2 cells: Involvement of ROS, Hsp70, JNK and ERK, and apoptosis. Molecules 20: 3758-3775, 2015.

35. Kim YK, Kim HJ, Kwon CH, Kim JH, Woo JS, Jung JS and Kim JM: Role of ERK activation in cisplatin-induced apoptosis in OK renal epithelial cells. J Appl Toxicol 25: 374-382, 2005.

36. Schafer $\mathrm{C}$ and Williams JA: Stress kinases and heat shock proteins in the pancreas: Possible roles in normal function and disease. J Gastroenterol 35: 1-9, 2000.

37. Napoli C, Cicala C, Wallace JL, de Nigris F, Santagada V, Caliendo G, Franconi F, Ignarro LJ and Cirino G: Proteaseactivated receptor-2 modulates myocardial ischemia-reperfusion injury in the rat heart. Proc Natl Acad Sci USA 97: 3678-3683, 2000.

38. Schmidlin F, Amadesi S, Dabbagh K, Lewis DE, Knott P, Bunnett NW, Gater PR, Geppetti P, Bertrand C and Stevens ME: Protease-activated receptor 2 mediates eosinophil infiltration and hyperreactivity in allergic inflammation of the airway. J Immunol 169: 5315-5321, 2002.

39. Ferrell WR, Lockhart JC, Kelso EB, Dunning L, Plevin R, Meek SE, Smith AJ, Hunter GD, McLean JS, McGarry F, et al: Essential role for proteinase-activated receptor-2 in arthritis. J Clin Invest 111: 35-41, 2003.

40. McLean PG, Aston D, Sarkar D and Ahluwalia A: Proteaseactivated receptor-2 activation causes EDHF-like coronary vasodilation: Selective preservation in ischemia/reperfusion injury: Involvement of lipoxygenase products, VR1 receptors, and C-fibers. Circ Res 90: 465-472, 2002.

41. Boyd JH, Mathur S, Wang Y, Bateman RM and Walley KR Toll-like receptor stimulation in cardiomyoctes decreases contractility and initiates an NF-kappaB dependent inflammatory response. Cardiovasc Res 72: 384-393, 2006.

42. Soh UJ, Dores MR, Chen B and Trejo J: Signal transduction by protease-activated receptors. Br J Pharmacol 160: 191-203, 2010.

43. Freedman NJ and Shenoy SK: Regulation of inflammation by $\beta$-arrestins: Not just receptor tales. Cell Signal 41: 41-45, 2018.

44. Patel PA, Tilley DG and Rockman HA: Physiologic and cardiac roles of beta-arrestins. J Mol Cell Cardiol 46: 300-308, 2009.

(i) $(-)$ This work is licensed under a Creative Commons Attribution-NonCommercial-NoDerivatives 4.0 International (CC BY-NC-ND 4.0) License. 